$07 ; 09 ; 13$

\title{
Лазерное, фотоэмиссионное возбуждение упругих колебаний в микрооптоэлектромеханических системах
}

\author{
(c) Ф.A. Егоров ${ }^{1,2}$ \\ ${ }^{1}$ НПК „Технологический центр“, Москва, Зеленоград, Россия \\ ${ }^{2}$ Фрязинский фрилиал Института радиотехники и электроники им. В.А. Котельникова РАН, Фрязино, \\ Московская обл., Россия \\ E-mail: egorov-fedor@mail.ru
}

Поступило в Редакцию 15 октября 2019г.

В окончательной редакции 2 апреля 2020 г.

Принято к публикации 6 апреля 2020г.

\begin{abstract}
Проанализированы возможность и эффективность лазерного возбуждения упругих колебаний в микрооптоэлектромеханических системах под действием силы отдачи при внешнем фотоэффекте. Показано, что в указанных системах на основе $\mathrm{GaAs}$ этот механизм возбуждения колебаний может оказаться на порядок более эффективным, чем давление света. Предложенный метод создания таких систем открывает возможности для разработки новых типов быстродействующих оптических функциональных элементов, управляемых с помощью света.
\end{abstract}

Ключевые слова: фотоэмиссия, микромеханический резонатор, сила отдачи, лазерное возбуждение, вакуумный диод, резонатор Фабри-Перо.

DOI: 10.21883/PJTF.2020.13.49590.18076

Квантовый выход внешнего фотоэффекта полупроводниковых структур на основе GaAs в ближнем ИК-диапазоне может достигать $Y \simeq 30 \%$ [1], что может приводить с учетом довольно узкого углового распределения фотоэлектронов $\theta \leqslant 10^{\circ}[2]$ к заметному импульсу отдачи, приобретаемому освещаемым участком поверхности, и, следовательно, к действию на этот участок фотоиндуцированной силы $(f)$. Поскольку GaAs (наряду с $\mathrm{Si}$, $\mathrm{Ge})$ является одним из основных материалов, используемых при создании микрооптомеханических резонансных систем (MOMRS) [3], представляется актуальным рассмотреть условия эффективного лазерного возбуждения акустомеханических колебаний MOMRS за счет фотоэмиссии, что определяет цель настоящей работы. Важной особенностью этого механизма возбуждения колебаний по сравнению с рядом других (фототермическим, фотопьезоэлектрическим и др. [4]) является безынерционность - ключевой фактор при возбуждении высокочастотных (наиболее интересных) MOMRS, открывающих новые возможности в области создания оптических устройств, управляемых светом.

На основе MOMRS, возбуждаемых лазерным излучением, возможно создание нелинейных резонаторов Фабри-Перо (NFPC), в которых взаимодействие между оптическими и механическими модами приводит к ряду интересных и важных эффектов [5]: преобразованию спектра оптического излучения; проявлениям квантовых свойств макрообъектов - MOMRS; трансформации теплового движения и динамическому охлаждению MOMRS. Это открывает новые уникальные возможности для исследований в таких областях, как, например, квантовая макрофизика, информатика, мезоскопика, а также позволяет создавать резонансные прецизионные датчики с миниатюрными чувствительными элементами на основе MOMRS. Исходя из этого представляется целесообразным исследовать эффективность фотоэмиссионного механизма возбуждения колебаний именно на примере NFPC, в котором MOMRS играет роль подвижного зеркала. Исследованный нами NFPC по существу представляет собой микрооптоэлектромеханическую систему (MOEMS), в основе которой лежит вакуумный диод (ВД) с фотокатодом (рис. 1). В данной системе роль фотокатода играет MOMRS (точнее, колебательный элемент (КЭ) MOMRS), а роль анода — полупрозрачный электрод (ППЭ), соединенный с MOMRS; они образуют внешнюю электрическую цепь, закорачивающую анод на фотокатод. Облучение КЭ лазерным излучением, вызывающим фотоэмиссию, осуществляется через ППЭ, эквипотенциальный с фотокатодом, при этом межэлектродное расстояние ВД одновременно является базой NFPC. Ограничимся для определенности случаем MOMRS с КЭ в виде микромембраны (рис. 1), отметив при этом, что изложенное далее в равной мере применимо и к другим типам КЭ (например, к микробалкам с консольным или двусторонним закреплением).

Полагаем, что начальная база NFPC - расстояние между зеркальными параллельными поверхностями КЭ и ППЭ с коэффициентами отражения $R$ и $R_{0}$ - является достаточно малой: $d_{0} \ll 2 a \ll D(D-$ диаметр микромембраны), что с учетом малого углового разброса скорости фотоэлектронов позволяет ограничиться одномерным приближением. Нелинейность NFPC обусловлена в основном зависимостью базы $d=d_{0}+s$ от смещений (деформаций) КЭ $s\left(F_{e}\right)$, вызванных фотоиндуцированной 


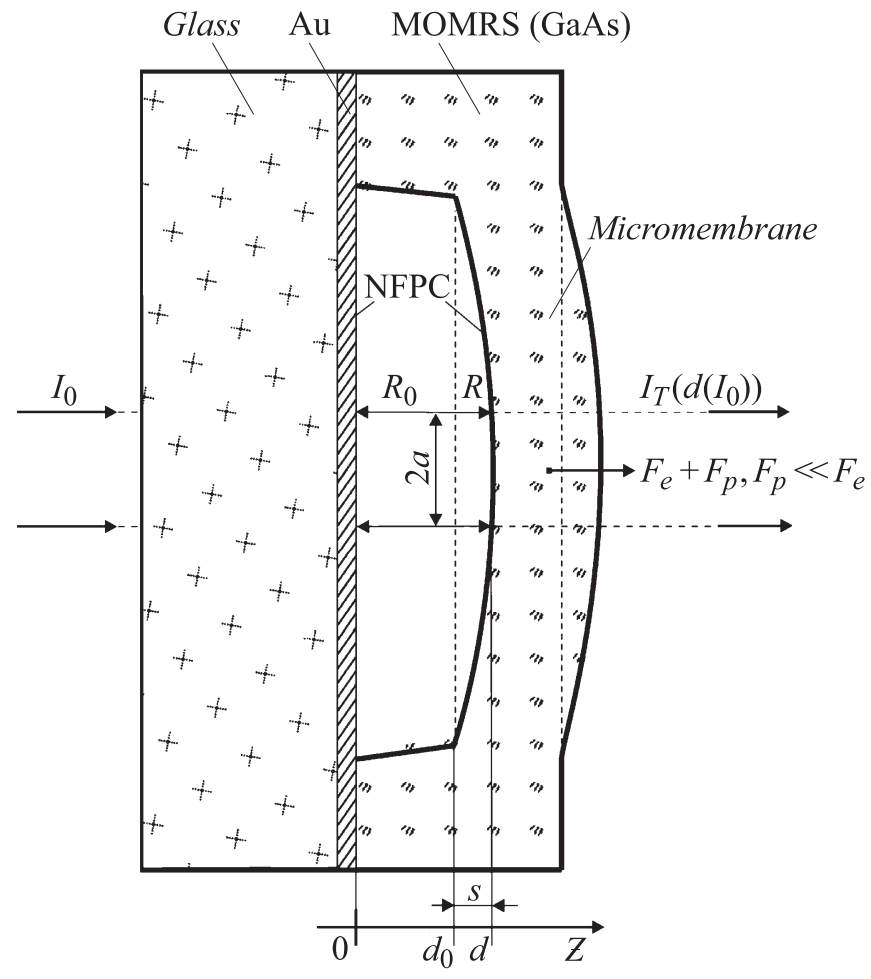

Рис. 1. Схема микрооптоэлектромеханической системы на основе MOMRS, облучаемой коллимированным когерентным (лазерным) пучком. NFPC - нелинейный резонатор Фабри-Перо, образованный отражающими поверхностями микромембраны (КЭ) и полупрозрачного электрода с соответствующими коэффициентами отражения $R$ и $R_{0}$.

силой $F_{e}\left(I_{0}\right)=p_{e} S: s\left(F_{e}\right) \approx 3\left(1-\gamma^{2}\right) D^{4} F_{e} / 16 \pi E h^{3}$, где $p_{e}$ - поверхностная плотность силы отдачи, действующей на КЭ с толщиной $h$, модулем Юнга $E$ и коэффициентом Пуассона $\gamma ; S=\pi a^{2}$ - площадь сечения коллимированного лазерного пучка. Микромембрана - фотокатод - может иметь достаточно сложную структуру, состоящую из ряда эпитаксиальных слоев GaAs с общей толщиной $h \approx 2-3 \mu \mathrm{m}$ [1], что обеспечивает практически полное поглощение ИК-излучения при $\lambda \leqslant 850 \mathrm{~nm}$. Полупрозрачный анод может представлять собой, например, стеклянную пластину, покрытую тонкой пленкой золота $(\mathrm{Au})$. Интенсивность излучения $(\mathrm{I})$, падающего на КЭ, описывается выражением [6]:

$$
I\left(I_{0}\right)=\left(1-R_{0}\right)\left[1+G^{2} \sin ^{2}\left(\frac{2 \pi d}{\lambda}\right)\right]^{-1}\left(1-\sqrt{R_{0} R}\right)^{-2} I_{0},
$$

где

$$
G=2 \sqrt{R_{0} R}\left(1-\sqrt{R_{0} R}\right)^{-1},
$$

$I_{0}-$ интенсивность когерентного (лазерного) излучения, падающего на MOEMS, $d=d_{0}+s\left(F_{e}\right)$. В peзультате интенсивность излучения, проходящего через MOEMS, имеет нелинейный характер:

$$
I_{T}\left(I_{0}\right)=(1-R) \eta I\left(I_{0}\right)
$$

где $\eta \ll 1-$ коэффициент пропускания фотокатода (КЭ). Максимальная нелинейность NFPC достигается в условиях „квадратуры“ интерферометра, реализующихся при значениях исходной базы $d_{k}=k \lambda / 4+\lambda / 8$, $k=1,2,3, \ldots$, которые при $k \gg 1$ обеспечивают необходимую оптическую добротность резонатора.

Фотоэлектронная эмиссия (рис. 2), несмотря на кажущуюся простоту единичного акта фотоэффекта, в условиях освещения поверхности тела интенсивным модулированным излучением имеет достаточно сложный характер $[7,8]$. В отличие от газа незаряженных частиц электронный газ способен удержаться и находиться вблизи внешней поверхности тела, так как в результате фотоэмиссии тело заряжается положительно и силы притяжения электронов к нему обусловливают наличие электронного облака с объемным зарядом (О3). Распределения основных величин - электрического потенциала $\varphi(z)$, потенциальной энергии $V(z)=e \varphi(z)$, скорости $v(z)$, плотности фотоэлектронов $n(z)$ и их потока $J(z)$ - в межэлектродном пространстве (в области О3) зависят от ряда факторов: характеристик фотокатода и оптического излучения с энергией фотонов $\varepsilon_{f}=h c / \lambda$, возбуждающих фотоэмиссию; конфигурации электродов (фотокатода, анода); граничных условий [7-9]. В стационарных режимах в силу закона сохранения энергии $k+V=k_{0}=$ const анода достигают лишь быстрые, $k_{0}>V_{m}$ фотоэлектроны, способные преодолеть потенциальный барьер $V_{m}$ (рис. $2, b$ ), которые создают электрический ток $\left(I_{a}\right)$ через ВД с плотностью потока фотоэлектронов вблизи анода $j_{a}=I_{a} / e S, e-$ заряд электрона; $k_{0}$ и $k-$ кинетическая энергия фотоэлектрона вблизи фотокатода и в области О3 соответственно.

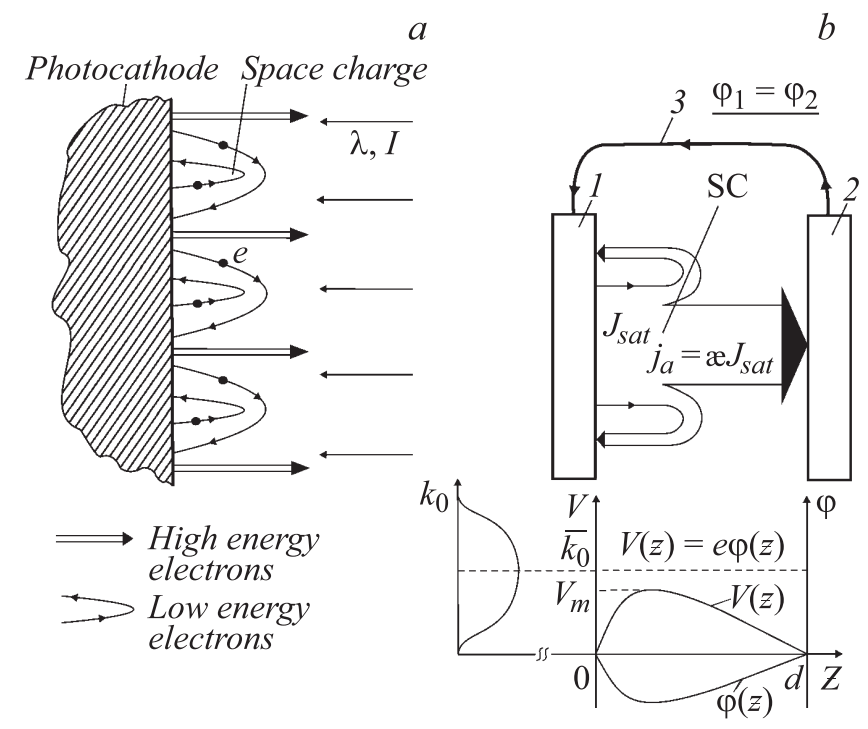

Рис. 2. $a-$ схема фрагмента фотокатода, освещаемого равномерно непрерывным оптическим излучением; $b-$ структура потоков фотоэлектронов и распределение (качественное) электрического потенциала в межэлектродном пространстве MOEMS: фотокатод (1), эквипотенциальный аноду (2), и электрический проводник (3). 
Движение остальной части фотоэлектронов является финитным: после отражения от потенциального барьера они возвращаются обратно на фотокатод (рис. 2, $a$ ) и, таким образом, не вносят вклада в ток через ВД. В связи с этим отметим существенную немоноэнергетичность фотоэлектронов, эмитируемых фотокатодом (GaAs) c работой выхода $A \approx 1.2 \mathrm{eV}[1,2]$, максимальная энергия $\left(k_{m}\right)$ и скорость $\left(v_{m}\right)$ которых, согласно закону Эйнштейна, составляют $k_{m}=m v_{m}^{2} / 2=\varepsilon_{f}-A(m-$ масса электрона). Плотность потока насыщения, составляющая $J_{s a t}=(1-R) Y I / \varepsilon_{f}$, в соответствии с уравнением неразрывности приводит к концентрации вблизи фотокатода $n_{0}=J_{s a t} / \bar{v}_{o z}$, где $\bar{v}_{0 z}=\left\langle v_{0 i} \cos \theta_{i}\right\rangle-z$-компонента средней скорости, причем $\bar{v}_{0}=\left\langle v_{0 i}\right\rangle \approx 0.6 v_{m}$. Ввиду малого углового разброса $\left(\theta_{i} \leqslant 0.2\right)$ полагаем $\bar{v}_{0 z} \approx \bar{v}_{0}$ (точность 2\%).

Результирующая поверхностная плотность силы $p_{e}=f_{z}+g_{z}$ состоит из двух слагаемых: $z$-компоненты плотности силы отдачи $f_{z}$, которая обусловлена суммарной плотностью импульса отдачи фотоэлектронов (с учетом финитного движения), и плотности силы $g_{z}=\sigma E_{0} / 2$, обусловленной электрическим полем О3 (с напряженностью $E_{0}$ ), действующим на фотокатод с поверхностной плотностью заряда $\sigma=\varepsilon_{0} E_{0}, \varepsilon_{0}-$ электрическая постоянная. Необходимые для расчетов $f_{z}, g_{z}$ величины $(n(z), \varphi(z), J(z)$ и др.) можно определить с помощью методов, разработанных в [7-9]. Однако изза сложного характера реальной функции распределения фотоэлектронов по скоростям (ФР) [1,2] это требует проведения сложных численных расчетов, которые в силу большого числа степеней свободы системы не позволяют в полной мере решить основную задачу установить аналитическую зависимость эффективности данного механизма возбуждения колебаний от основных параметров системы. В связи с этим нами предложен упрощенный способ оценки силы отдачи $F_{e}$, основанный на приближенном рассмотрении тока через ВД в виде череды отдельных актов переноса дискретных порций зарядов $\left(q_{k}\right)$ - сгустков фотоэлектронов с фотокатода на анод. Эти переносы происходят благодаря „инерционному“ движению фотоэлектронов, вызванному их начальным импульсом, приводящим к импульсу силы отдачи $f_{z k}$. Если период импульсов $T>\tau_{r e l}\left(\tau_{r e l}-\right.$ время релаксации в MOEMS), то после каждого отдельного акта переноса заряда система возвращается в исходное состояние без какого-либо О3 в межэлектродном пространстве. Важно отметить, что анода достигает лишь часть порций зарядов - быстрые фотоэлектроны, способные преодолеть потенциальный барьер, созданный зарядами-,,изображениями“, индуцированными на электродах при прохождении порций зарядов в межэлектродном пространстве. Средняя сила отдачи $F_{e}=\left\langle f_{z k}\right\rangle$, действующая на облучаемый участок КЭ, определяется полным потоком импульса, переносимым фотоэлектронами на анод: $F_{e}=P_{e}=\left\langle j_{a i} \mathbf{m} v_{a i}\right\rangle S$, где $j_{a i}=n_{a i} v_{a i}-$ плотность потока $i$-й группы электронов, достигающих анода со скоростью $v_{a i}$.
Замена $v_{a i}$ на $\bar{v}_{0}$ с учетом соотношения для средних скоростей $\bar{v}_{a i}>\bar{v}_{0}$ приводит к оценке снизу для фотоиндуцированной силы: $F_{e}>F_{\min }=I_{a} m \bar{v}_{0} / e$, при этом задача сводится к расчету максимального тока через ВД подробно рассмотренному в работе [9]. С учетом существенной немоноэнергетичности фотоэлектронов $\left|v_{0 i}-\bar{v}_{0}\right| / \bar{v}_{0} \leqslant 0.3$ и результатов численных исследований $[10]$, получим $I_{a} \approx \varkappa I_{\mathrm{SCL}}$, где $I_{\mathrm{SCL}}-$ порог Бурсиана для пучка с параметрами $\bar{v}_{0}, J_{s a t}, n_{0} ; \varkappa \leqslant 1-$ коэффициент, зависящий от свойств ФР. При оптимальных $d_{0}$ и $\lambda$, определяемых из соотношений $d_{0}=4 L_{D} / 3=25 \lambda / 8$ (где $L_{D}=\left(2 \varepsilon_{0} \bar{k}_{0} / n_{0} e^{2}\right)^{1 / 2}-$ пучковая длина дебаевской экранировки [9]), с учетом $\left|v_{0 i}-\bar{v}_{0}\right| / \bar{v}_{0} \leqslant 0.3$ получим оценку величины $\chi \simeq 0.4$. Используя формулу для силы давления света на фотокатод $F_{p}=(1+R) I S / c$ (сонаправленной с $F_{e}$ ), выражение для фотоиндуцированной силы можно представить в виде

$$
F_{\min }=(1-R) \varkappa Y m c \bar{v}_{0}\left((1+R) \varepsilon_{f}\right)^{-1} F_{p} .
$$

Для GaAs-фотокатодов с характеристиками $Y=30 \%$, $R=0.3 \quad[1,2]$ при допустимых параметрах MOEMS $\left(I_{0}=1.5 \cdot 10^{6} \mathrm{~W} / \mathrm{m}^{2}, \varepsilon_{f}=1.5 \mathrm{eV}(\lambda \approx 820 \mathrm{~nm}), R_{0}=0.5\right.$, $\left.a=20 \mu \mathrm{m}, I_{0} S \approx 2 \mathrm{~mW}\right)$ в соответствии с (2) получим $F_{\min } \approx 15 F_{p}$, откуда следует, что фотоэлектронная эмиссия в качестве механизма возбуждения колебаний может быть значительно более эффективной, чем давление света: $F_{e} \gg F_{p}$. Стационарное значение $F_{e}$ может быть использовано также в случае модулированного излучения, если длительность импульсов $\tau>\tau_{\text {rel }} \simeq\left(\tau_{0}^{2}+\tau_{o p t}^{2}+\tau_{R C}^{2}\right)^{1 / 2}$, где $\tau_{\text {opt }}, \tau_{R C}-$ время установления оптической моды в NFPC и длительность переходных процессов во внешней цепи с фотокатодом и анодом, которые уподобляются конденсатору, $\tau_{0} \simeq \sigma / e J_{\text {sat }} \approx 2 L_{D} / \bar{v}_{0}$ - время формирования ОЗ вблизи фотокатода. В MOEMS с ППЭ в виде тонкой $(\sim 10 \mathrm{~nm})$ пленки золота с сопротивлением между центрами фотокатода и анода $\sim 10 \Omega$ (при $D=1 \mathrm{~mm}$ ) имеем $\tau_{\text {opt }} \ll \tau_{R C} \leqslant \tau_{0}$, откуда $\tau_{\text {rel }} \approx \tau_{0} \approx 20$ ps. В результате верхняя граничная частота колебаний силы $F_{e}$ может достигать $f_{\max } \approx \frac{1}{2 \tau_{\text {rel }}} \simeq 25 \mathrm{GHz}$.

В заключение отметим следующее: с учетом того, что ВД по существу представляет собой колебательную систему [11], свойства MOEMS определяются взаимодействием трех существенно разных по своей природе резонаторов: электронного (ВД); оптического (NFPC) и механического (MOMRS), что может приводить к интересной нелинейной динамике, зависящей от соотношений между их собственными частотами. Это открывает новые возможности для создания на основе MOEMS быстродействующих оптических функциональных элементов (модуляторов, переключателей и др.), управляемых оптическим излучением.

\section{Финансирование работы}

Работа выполнена в рамках Госзаказа. 


\section{Конфликт интересов}

Автор заявляет, что у него нет конфликта интересов.

\section{Список литературы}

[1] Zhang Y., Jiao G. // Advances in photodetectors - research and applications. IntechOpen, 2019. Ch. 4.

DOI: 10.5772/intechopen.80704

[2] Karkare S., Dimitrov D., Schaff W., Cultrera L., Bartnik A., Liu X., Sawyer E., Esposito T., Bazarov I. // J. Appl. Phys. 2013. V. 113. P. 104904.

[3] Springer handbook of nanotechnology / Ed. B. Bhushan. 4th ed. Berlin-Heidelberg: Springer-Verlag, 2017. $1671 \mathrm{p}$.

[4] Okamoto H., Watanabe T., Ohta R., Onomitsu K., Gotoh H., Sogawa T., Yamaguchi H. // Nature Commun. 2015. V. 6. P. 8478. DOI: $10.1038 /$ ncomms 9478

[5] Cavity optomechanics. Nano- and micromechanical resonators interacting with light / Eds M. Aspelmeyer, T.J. Kippenberg, F. Marquardt. Berlin-Heidelberg: Springer-Verlag, 2014. $353 \mathrm{p}$.

[6] Борн М., Вольф Э. Основы оптики. М.: Наука, 1970. 856 с.

[7] Добрецов Л.Н., Гомоюнова М.В. Эмиссионная электроника. М.: Наука, 1966. 564 с.

[8] Резников Б.И., Субашиев А.В. // ЖТФ. 2002. Т. 72. В. 5. C. $95-101$.

[9] Кузнецов В.И., Эндер А.Я. // ЖТФ. 2013. Т. 83. В. 12. С. $1-$ 10.

[10] Алешин И.М., Кузьменков Л.С. // Вестн. МГУ. Сер. 3. Физика, астрономия. 1994. Т. 35. № 2. С. 46-50.

[11] Kuznetsov V.I., Gerasimenko A.B. // J. Appl. Phys. 2019. V. 125. P. 183301. 